\title{
Anti-Obesity Effects of a Mixture of Fermented Ginseng, Bifidobacterium longum BORI, and Lactobacillus paracasei CH88 in High-Fat Diet-Fed Mice
}

\author{
Dayoung Kang ${ }^{1}$, Zhipeng $\mathrm{Li}^{2}$, and Geun Eog $\mathrm{Ji}^{1,3 *}$ \\ ${ }^{1}$ Department of Food and Nutrition, Research Institute of Human Ecology, Seoul National University, Seoul 08826, Republic of Korea \\ ${ }^{2}$ Department of Biomedical Science, College of Veterinary Medicine, Oregon State University, Corvallis, OR 97331-4801, USA \\ ${ }^{3}$ Research Institute, Bifido Co., Ltd., Hongchun 25117, Republic of Korea
}

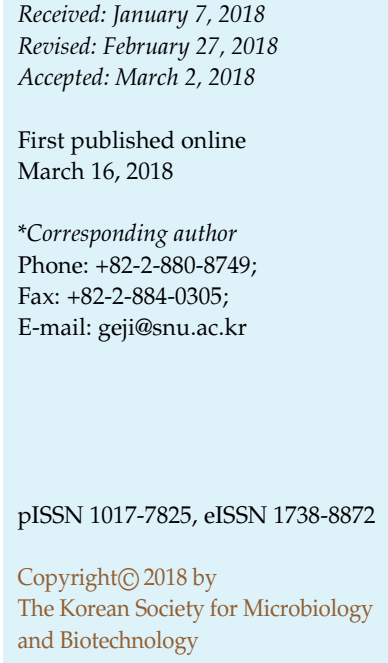

Ginseng and probiotics have anti-obesity effects in mice fed a high-fat diet (HFD). Absorption of ginsenoside and colonization of probiotics occur in the intestine. In this study, a mixture of fermented ginseng and two probiotics, Bifidobacterium longum BORI and Lactobacillus paracasei $\mathrm{CH} 88$, was administered to HFD-fed mice for 9 weeks. The mixture significantly suppressed weight gain $(p<0.05, n=8)$ and lipid deposition in the liver and adipose tissues as well as increased the mice's food intake. The adipocyte size of the adipose tissue was significantly decreased in the mixture-fed group, especially when $0.5 \%$ fermented ginseng and $5 \times 10^{8} / \mathrm{ml}$ of the two probiotics were used $(p<0.05, n=10)$. The expression of TNF- $\alpha$ in adipose tissue was efficiently downregulated in the mixture-fed group $(p<0.05, n=4)$. The supplement also improved the mice's fasting blood glucose levels $(p<0.05, n=8)$ and total cholesterol feces excretion $(p<0.05, n=8)$. The mixture of fermented ginseng and B. longum BORI and L. paracasei $\mathrm{CH} 88$ could have an anti-obesity effect and suppress lipid deposit in the liver and adipose tissues.

Keywords: Ginseng, lactobacteria, bifidobacteria, anti-obesity, lipid metabolism

\section{Introduction}

Ginseng has been used as an anti-obesity treatment in Korea and China for many years. The physiological benefits of ginseng and various ginsenosides have been proven by several studies. Jung et al. [1] reported that ginseng fed to rats with a high-fat diet (HFD) resulted in weight loss, reduced adiposity, and decreased hepatic triacylglycerol. Meanwhile, because the public's interest in probiotics has recently increased, much research has been conducted on probiotics. Probiotics are living organisms that confer beneficial impacts on the maintenance of the host health by acting on the gut microbiota [2]. The ingestion of Lactobacillus and Bifidobacterium has been proven to aid against obesity, inflammation, and related metabolic diseases [3]. Bifidobacterium longum BORI and Lactobacillus paracasei CH88 were industrially developed and various health benefits have been reported. B. longum BORI has novel probiotic features, and the frequency of diarrhea was significantly reduced in rotavirus-infected children with acute colitis [4]. B. longum BORI demonstrated the highest anti-obesity effect on weight loss as well as inhibited lipid deposition in the liver of HFD-fed mice [5]. Thus, both ginseng and probiotics are effective anti-obesity treatments.

In recent years, there has been a growing demand among consumers for probiotics and probiotic foods. Ginseng absorption and probiotic colonization are closely related and occur in the intestine [6-8]. The pharmacological action of ginsenosides occurs via biotransformation, which is deglycosylation by intestinal bacteria [9]. According to $\mathrm{Ku}$ [6], fermented ginseng $(\mathrm{FG})$ is a product that contains a significant amount of biocatalized ginsenoside glycosides via microbial glycosyl hydrolase treatment. When biotransformation occurs, ginsenoside becomes relatively hydrophobic, which improves their bioavailability by the body absorbing them. Meanwhile, probiotics alter the 
composition of the intestinal microflora via attachment and colonization of the intestinal mucosa, resulting in healthful effects [10]. However, few studies have researched the mechanism of FG and probiotics when consumed together.

Therefore, the purpose of this study was to evaluate whether a FG and probiotics mixture had an anti-obesity effect on mice fed a HFD.

\section{Materials and Methods}

\section{Fermented Ginseng and Probiotics}

The FG and probiotics used in this study were in freeze-dried powder form and provided by Bifido Co., Ltd (Korea). The probiotics mix (PM) was prepared by mixing Bifidobacterium longum BORI and Lactobacillus paracasei $\mathrm{CH} 88$ at a ratio of 1:1.

\section{Ginsenoside Analysis of Fermented Ginseng}

To determine the ginsenoside content, the FG was ultrasonically extracted with $80 \%$ methanol for $1 \mathrm{~h}$. The pre-treated samples were analyzed using an Ultimate 3000 HPLC system (Thermo Dionex, USA) and $210 \mathrm{~nm}$ UV detector. A VSD C-18 column $(250 \times 4.6 \mathrm{~mm}, 5 \mu \mathrm{m})$ was used for all separations. The mobile phase solvent consisted of distilled water (A) and acetonitrile (B). The gradient elution conditions were as follows: $0-50 \mathrm{~min}(5 \% \mathrm{~B})$, $50-56 \mathrm{~min}(95 \% \mathrm{~B})$, and $56-60 \mathrm{~min}(5 \% \mathrm{~B})$. The flow rate and temperature were set to $1 \mathrm{ml} / \mathrm{min}$ and $20^{\circ} \mathrm{C}$, respectively. Ginsenosides $\mathrm{Rb} 1, \mathrm{Rb} 2, \mathrm{Rd}, \mathrm{Rg} 1$, and F1 were purchased from Biotech (China). Ginsenosides Rc, Re, Rg2, Rg3, Rh1, Rh2, and cK were obtained from Cogon Biotech (China).

\section{Animals and Diets}

In our study, male ICR mice (7 weeks old) were purchased from Central Lab. Animal (Korea). The animal breeding environment was adjusted to a cycle of $12 \mathrm{~h}$ light $/ 12 \mathrm{~h}$ dark at a temperature of $23 \pm 1{ }^{\circ} \mathrm{C}$ and a humidity of $40-60 \%$. After a 1-week adaptation period, the mice were randomly divided into the following five groups $(n=8)$ : LFD (Low-fat diet; $10 \%$ fat of total calories), HFD (High-fat diet; $60 \%$ fat of total calories), or HFD supplemented with FG and PM (FG+PM) divided into LOW $\left(0.25 \% \mathrm{FG}+2.5 \times 10^{8}\right.$ $\mathrm{CFU} / \mathrm{ml} \mathrm{PM}), \mathrm{MID}\left(0.5 \% \mathrm{FG}+5 \times 10^{8} \mathrm{CFU} / \mathrm{ml} \mathrm{PM}\right)$, and $\mathrm{HIGH}$ $\left(1.0 \% \mathrm{FG}+1 \times 10^{9} \mathrm{CFU} / \mathrm{ml} \mathrm{PM}\right)$.

The mice were fed the experimental diets for 9 weeks, following which they were anesthetized with Zoletil (Virbac Lab., France) and Rompun (Bayer, Germany) after fasting for $12 \mathrm{~h}$. Cardiac blood, liver tissue, epididymal fat, and mesenteric fat were removed from the specimens and weighed. All procedures were approved by the Institutional Animal Care and Use Committee (IACUC; SNU-161108-7) (Seoul National University, Korea).

\footnotetext{
Histological Analysis

Morphological analysis of the liver and epididymal fat was performed to measure the hepatic fat accumulation and adipocyte
}

size in the adipose tissue (AT). The liver and epididymal fat were fixed in 10\% formalin (Wako Pure Chemical Industries, Japan) for 1 day and then stored in paraffin. Sections were then cut, stained with hematoxylin and eosin, and examined with an optical microscope.

\section{Biochemical Analysis}

The plasma triacylglycerol (TAG), total cholesterol (TC), and high-density lipoprotein cholesterol (HDL-C) were analyzed using a kit purchased from Asanpharm (Korea). The low-density lipoprotein cholesterol (LDL-C) was calculated by subtracting HDL-C from TC. The fasting blood glucose (FBG) was measured after $12 \mathrm{~h}$ of fasting in the 9th week. The plasma insulin was analyzed using a mouse insulin ELISA kit (AKRIN-011T; Shibayagi, Japan).

\section{Hepatic Lipid Analysis}

According to Li et al. [5], we used a modified Folch method [11] for the liver lipid analysis. The liver lipid was measured using a TAG test kit (Asanpharm, Korea).

RNA Extraction and Quantitative Real-Time Reverse Transcriptase Polymerase Chain Reaction (RT-PCR)

The total RNAs of the liver and AT were extracted using a TaKaRa MiniBEST Universal RNA Extraction kit (TaKaRa Bio, Japan) and RNeasy Lipid Tissue Mini Kit (Qiagen, Germany), respectively. The RNA was reverse transcribed to cDNA using a cDNA kit from TaKaRa Bio. mRNA expression analysis of the cDNA and SYBR premix Ex Taq (TaKaRa Bio, Japan) was performed with a StepOne real-time PCR system. The analysis conditions were denaturation at $95^{\circ} \mathrm{C}$ for $30 \mathrm{sec}$, followed by 40 cycles of $95^{\circ} \mathrm{C}$ for $5 \mathrm{sec}$ and $60^{\circ} \mathrm{C}$ for $33 \mathrm{sec}$. The data were nomalized for the housekeeping gene GAPDH and compared with the expression levels of the HFD group.

\section{TC in Feces}

The collected feces was ground into a powder and the subsequent process was the same as for the hepatic lipid analysis. The TC in feces was measured using a TC test kit (Asanpharm, Korea).

\section{Statistical Analysis}

The results were analyzed using the SPSS statistical package ANOVA test. The statistical differences were examined via Duncan's multiple range test. The statistical significance was set at $p<0.05$.

\section{Results}

\section{Effect of FG+PM on Body Weight and Food Intake}

The main ginsenosides of the experimental FG were protopanaxadiol-type $\mathrm{Rd}(19.1 \%), \quad \mathrm{Rc} \quad(4.5 \%)$, and protopanaxatriol-type $\operatorname{Re}(3.3 \%)$. The others were $\mathrm{Rb} 1$ 
Table 1. Effects of fermented ginseng plus probiotic mixtures on weight parameters of mice.

\begin{tabular}{lccccc}
\hline & LFD & HFD & LOW & MID & HIGH \\
\hline Initial BW (g) & $38.6 \pm 1.1$ & $38.9 \pm 1.4$ & $37.4 \pm 0.7$ & $38.2 \pm 1.9$ & $37.6 \pm 1.9$ \\
Final BW (g) & $52.1 \pm 5.3^{\mathrm{a}}$ & $67.2 \pm 6.2^{\mathrm{c}}$ & $65.1 \pm 8.9^{\mathrm{bc}}$ & $58.0 \pm 8.6^{\mathrm{ab}}$ & $65.0 \pm 7.1^{\mathrm{bc}}$ \\
BW gain (g) & $13.5 \pm 4.4^{\mathrm{a}}$ & $28.4 \pm 5.6^{\mathrm{b}}$ & $27.8 \pm 8.5^{\mathrm{b}}$ & $19.8 \pm 8.1^{\mathrm{a}}$ & $27.4 \pm 7.2^{\mathrm{b}}$ \\
Food intake (kcal) & $3,589.0 \pm 29.3^{\mathrm{a}}$ & $4,641.0 \pm 1,150.5^{\mathrm{b}}$ & $5,671.2 \pm 179.5^{\mathrm{c}}$ & $6,021.8 \pm 493.3^{\mathrm{c}}$ & $5,879.9 \pm 1,260.2^{\mathrm{c}}$ \\
Food efficiency $^{1}$ & $0.0038 \pm 0.001^{\mathrm{a}}$ & $0.0064 \pm 0.002^{\mathrm{b}}$ & $0.0049 \pm 0.002^{\mathrm{ab}}$ & $0.0034 \pm 0.002^{\mathrm{a}}$ & $0.0048 \pm 0.002^{\mathrm{ab}}$ \\
Liver $_{\text {EAT }^{2}}^{1.9 \pm 0.24^{\mathrm{a}}}$ & $2.9 \pm 0.77^{\mathrm{b}}$ & $2.4 \pm 0.66^{\mathrm{ab}}$ & $2.2 \pm 0.75^{\mathrm{a}}$ & $2.5 \pm 0.44^{\mathrm{ab}}$ \\
MAT $^{3}$ & $2.0 \pm 0.65^{\mathrm{a}}$ & $3.1 \pm 0.60^{\mathrm{bc}}$ & $3.3 \pm 0.90^{\mathrm{c}}$ & $2.5 \pm 0.88^{\mathrm{ab}}$ & $3.0 \pm 0.52^{\mathrm{bc}}$ \\
\hline
\end{tabular}

${ }^{1}$ Food efficiency $=$ food intake $(\mathrm{kcal}) / \mathrm{BW}$ gain $(\mathrm{g})$.

${ }^{2}$ EAT, Epididymal adipose tissue.

${ }^{3}$ MAT, Mesenteric adipose tissue.

${ }^{\text {abcd }}$ Means in the same row not sharing a common letter are significantly different at $p<0.05(n=8)$.

(1.4\%), Rb2 (0.7\%), Rg1 (0.9\%), Rg2 (0.7\%), and Rg3 (1.0\%). Table 1 shows the changes in mouse body weight and food consumption. The mice's weight at the 9th week was significantly increased in the HFD group compared with the LFD group. Compared with the control group, the MID group showed a significant decrease in body weight and weight gain.

The HFD group had a significant increased food intake compared with the LFD group, and the experimental groups consumed more calories than did the control. The food efficiency of the MID group was significantly lower than that of the HFD group.

\section{Effect of FG+PM on the Weights of the Liver, Epididymal} Adipose Fat, and Mesenteric Adipose Fat

Table 1 shows the liver and the epididymal and mesenteric AT (EAT and MAT) weights. The liver weight was significantly decreased in the MID group compared with the control group. The EAT and MAT weights were significantly increased in the HFD group compared with the LFD group.

\section{Effect of FG+PM on the Fasting Blood Glucose and Insulin Levels}

The HFD group had a higher FBG level than that of the LFD group. The FBG levels of the experimental groups were lower than those of the HFD group, and those of the MID group were significantly lower (Fig. 1A). Although the MID group had the lowest insulin level, there was no significant difference (Fig. 1B).

\section{Effect of FG+PM on the Plasma Lipid Profiles}

As shown in Table 2, the mice fed the FG+PM had similar plasma TAG levels. Although the plasma TC and LDL-C
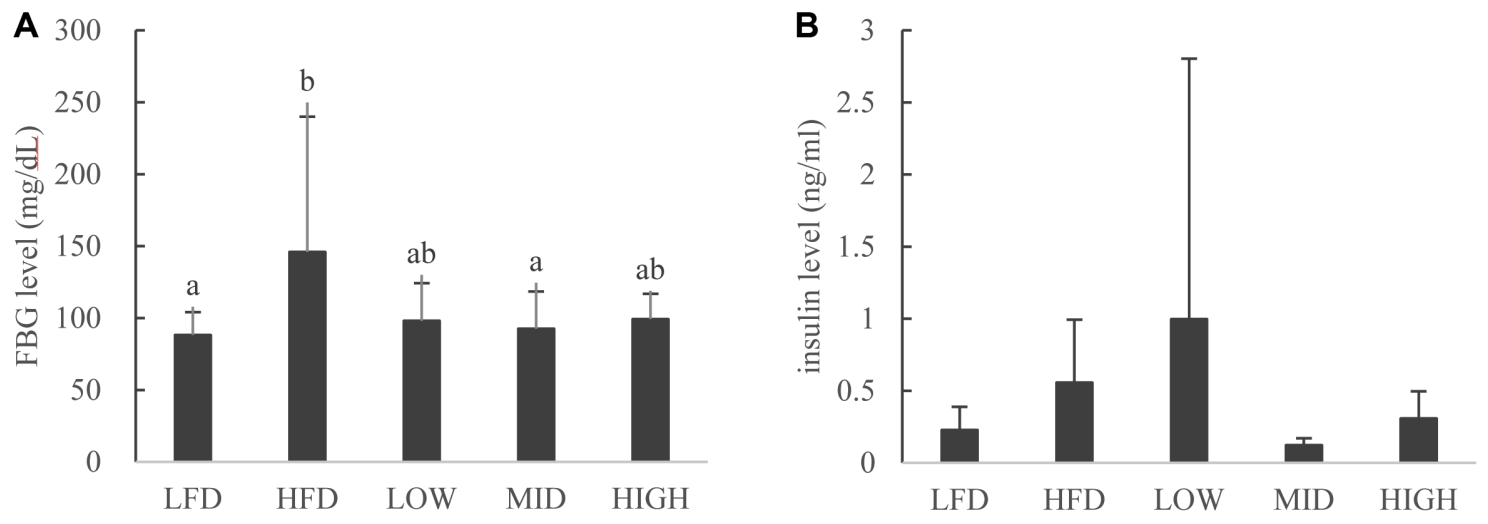

Fig. 1. Effects of fermented ginseng plus probiotic mixtures on fasting blood glucose (FBG) and insulin levels.

(A) Plasma FBG levels $(n=8)$. (B) Plasma insulin levels $(n=7)$. ${ }^{\text {abcd }}$ Means not sharing a common letter are significantly different at $p<0.05$. 
Table 2. Effects on FG+PM on the plasma lipid profile.

\begin{tabular}{lccccc}
\hline & LFD & HFD & LOW & MID & HIGH \\
\hline TAG $(\mathrm{mg} / \mathrm{dl})$ & $27.3 \pm 9.7$ & $19.4 \pm 9.8$ & $24.5 \pm 10.8$ & $20.2 \pm 4.3$ & $22.2 \pm 8.5$ \\
TC $(\mathrm{mg} / \mathrm{dl})$ & $125.5 \pm 9.4^{\mathrm{c}}$ & $109.2 \pm 9.3^{\mathrm{b}}$ & $91.2 \pm 11.4^{\mathrm{a}}$ & $138.3 \pm 18.7^{\mathrm{d}}$ & $123.5 \pm 6.3^{\mathrm{c}}$ \\
LDL-C $(\mathrm{mg} / \mathrm{dl})$ & $75.6 \pm 7.3^{\mathrm{c}}$ & $62.6 \pm 6.6^{\mathrm{b}}$ & $51.0 \pm 7.1^{\mathrm{a}}$ & $98.4 \pm 13.6^{\mathrm{d}}$ & $82.5 \pm 9.0^{\mathrm{c}}$ \\
HDL-C $(\mathrm{mg} / \mathrm{dl})$ & $49.8 \pm 4.1^{\mathrm{b}}$ & $46.5 \pm 8.3^{\mathrm{ab}}$ & $40.2 \pm 6.3^{\mathrm{a}}$ & $39.9 \pm 12.8^{\mathrm{a}}$ & $41.0 \pm 9.3^{\mathrm{ab}}$ \\
\hline
\end{tabular}

${ }^{\text {abd }}$ Means in the same row not sharing a common letter are significantly different at $p<0.05(n=8)$.

levels of the LOW group were significantly lower than those of the HFD group, the others were significantly higher. The plasma HDL-C levels of the experiment groups were lower than those of the HFD group; however, there

A

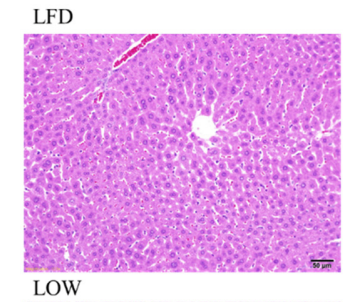

HFD
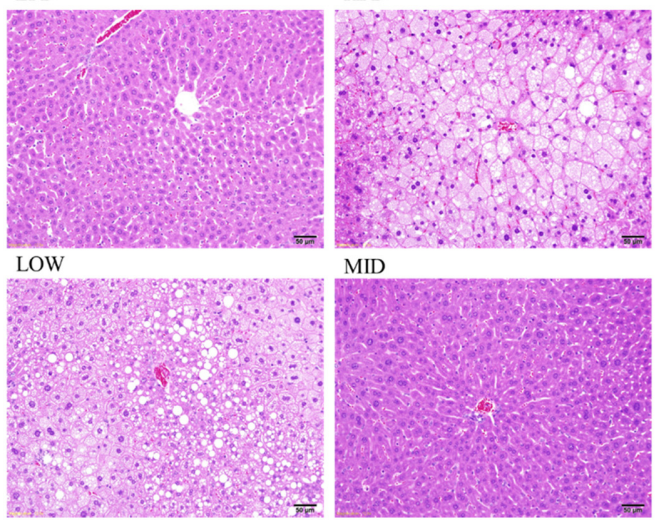

MID

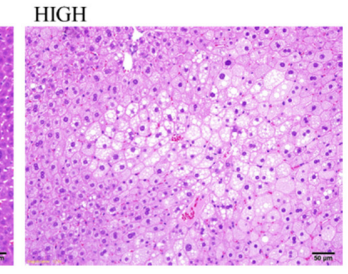

B

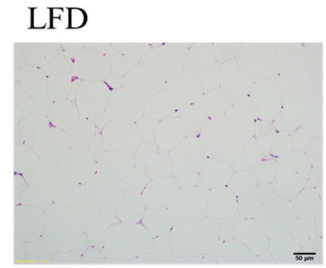

HFD
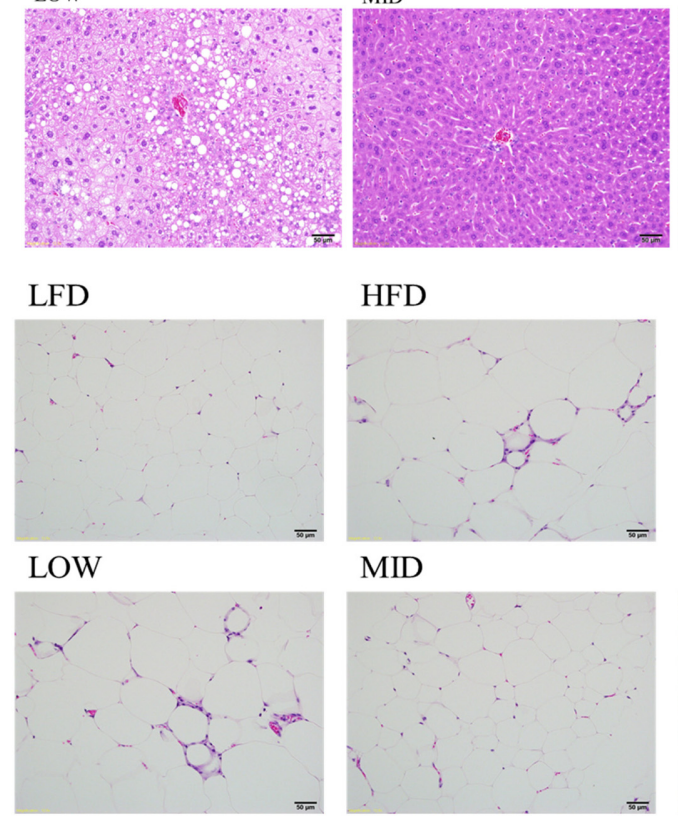

MID

HIGH
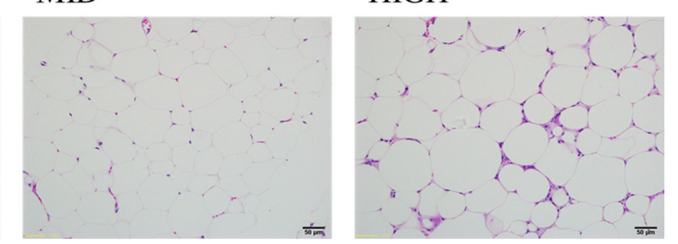

C

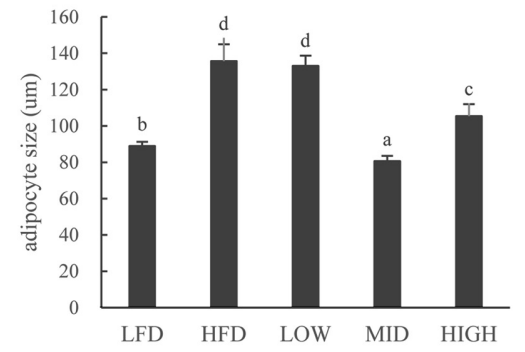

Fig. 2. Effects of fermented ginseng plus probiotic mixtures on the histology of liver and adipose tissue.

(A) H\&E staining of liver. (B) H\&E staining of adipose tissue. (C) Adipocyte size of adipose tissue. abcdMeans not sharing a common letter are significantly different at $p<0.05$. 


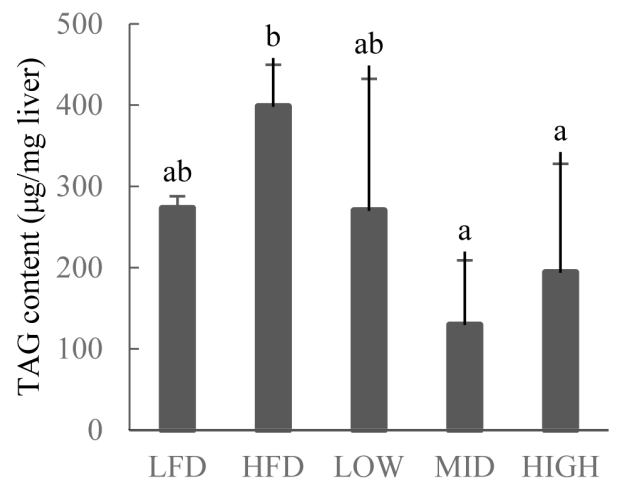

Fig. 3. Effects of fermented ginseng plus probiotic mixtures on the TAG content in the liver $(n=4)$.

${ }^{\text {abcd }}$ Means not sharing a common letter are significantly different at $p<0.05$.

was no significant difference.

\section{Effect of FG+PM on the Histology of the Liver and AT}

The lipid accumulations in the liver and AT were determined by H\&E staining. The HFD group had higher lipids than the LFD group, and the lowest lipid accumulation of the experimental groups was in the MID group (Fig. 2A). The adipocyte sizes in the EAT were confirmed (Fig. 2B). The HFD group had a significantly larger adipocyte size than that of the LFD group, and the adipocyte size of the MID and HIGH groups were significantly smaller than that of the HFD group. (Fig. 2C)

\section{Effect of FG+PM on the Hepatic Lipid Profiles}

The TAG content was lower in the experimental group than in the HFD group, and the lowest in the MID group (Fig. 3).

\section{Effect of FG+PM on the mRNA Expression in Liver Tissue}

Figs. $4 \mathrm{~A}$ and $4 \mathrm{~B}$ show the mRNA expression of the lipids and glucose metabolism in the liver. The expression of peroxisome proliferator-activated receptor- $\gamma$ (PPAR- $\gamma$ ) was significantly higher in the HFD group than in the LFD group. The expression of LDL receptor (LDLR) was significantly higher in the LOW group than in the control group. The HFD group had a significantly higher expression of glucokinase (GK) than that of the LFD group.

\section{Effect of FG+PM on the mRNA Expression in AT}

Fig. 5A shows the mRNA expression of AT inflammation. The expression of tumor necrosis factor (TNF)- $\alpha$ was
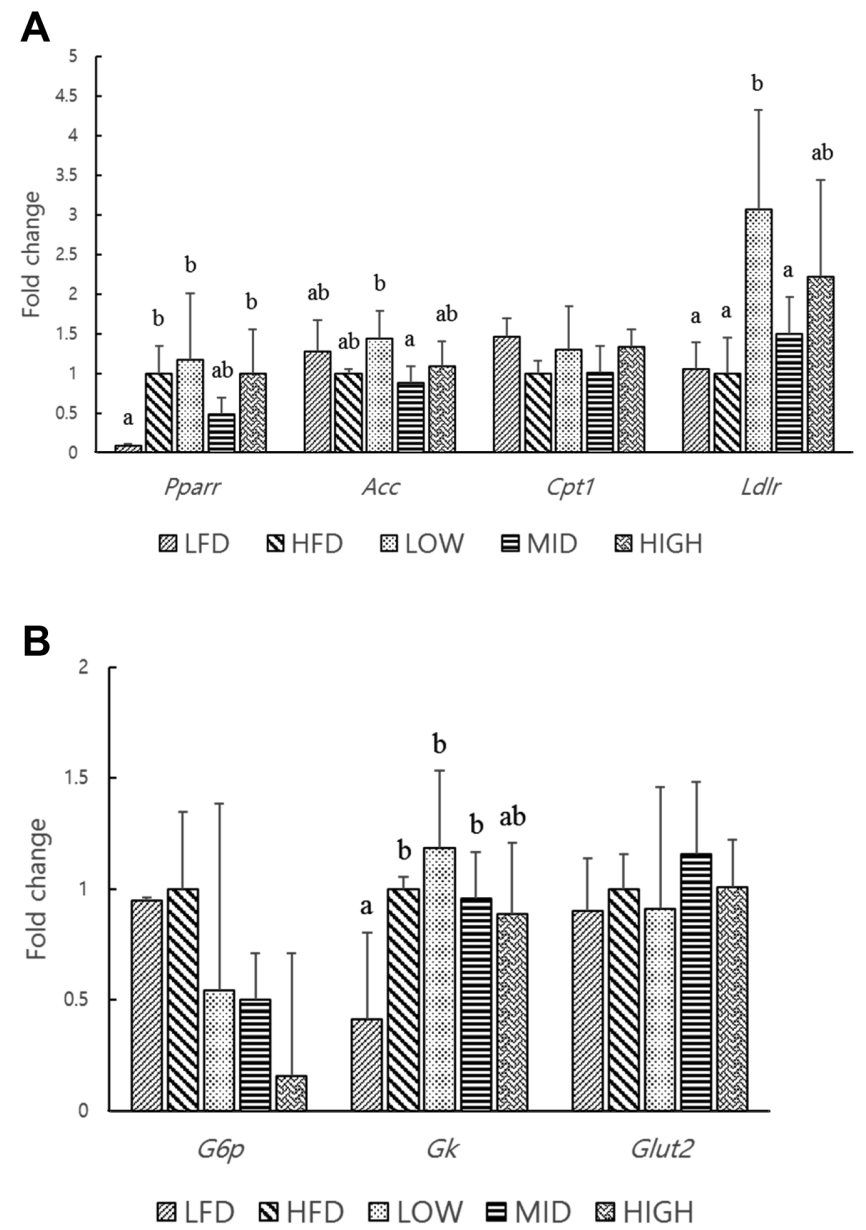

Fig. 4. Effects of fermented ginseng plus probiotic mixtures on mRNA expression in liver.

(A) Effects on mRNA expression related to lipid metabolism $(n=4)$. (B) Effects on mRNA expression related to glucose metabolism $(n=4)$. ${ }^{\text {abcd }}$ Means not sharing a common letter are significantly different at $p<0.05$.

significantly higher in the HFD group than in the LFD group and significantly lower in the MID and HIGH groups than in the control group.

Fig. 5B shows the mRNA expression of AT lipid metabolism. The expression of PPAR- $\gamma$ was increased in the MID group compared with the control; however, there was no significant difference. The expression of lipoprotein lipase (LPL) in the MID group was higher than that in the control group but not significant.

\section{Effect of FG+PM on the TC in Feces}

The TC content in the plasma was significantly higher in the HFD group than in the LFD group. The TC content in 
A

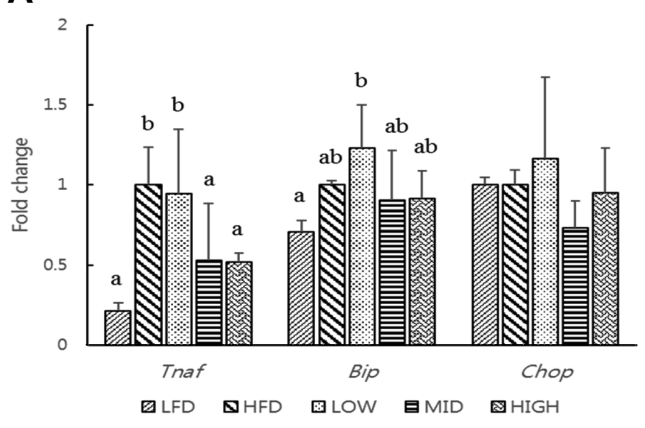

B

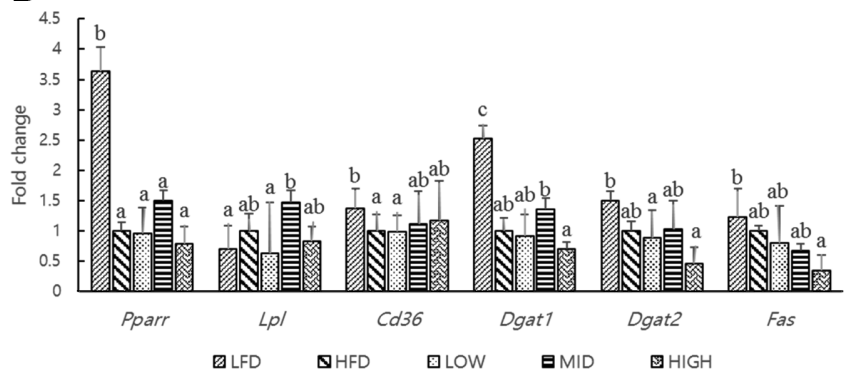

Fig. 5. Effects on fermented ginseng plus probiotic mixtures on mRNA expression in adipose tissue.

(A) Effects on mRNA expression related to inflammation $(n=4)$. (B) Effects on mRNA expression related to lipid metabolism $(n=4)$.

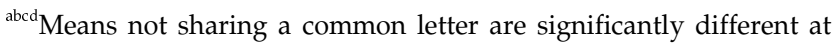
$p<0.05$.

the feces was higher in all the FG+PM groups than in the control group, and the LOW and HIGH groups had a significantly higher TC feces content (Fig. 6).

\section{Discussion}

It has been reported that a HFD induces obesity and impairs leptin and insulin signaling in the hypothalamus, which results in excessive weight gain [12]. Liu et al. [13] reported that the ginsenosides $\mathrm{Rd}, \mathrm{Rb} 1, \mathrm{Rb} 2$, and $\mathrm{Rc}$ are pancreatic lipase inhibitors that prevent obesity by increasing fat excretion. Similarly, probiotics have reduced fat accumulation and weight gain by competing with other probiotic strains in the intestines for nutrients and by reducing the gut microbial diversity [14]. The results of our study are similar to those found in earlier experimental studies. The intake of FG+PM reduced weight gain, especially in the MID group, and significantly suppressed weight gain despite increased food intake.

Obesity is associated with dyslipidemia, which increases TAG and free fatty acids (FFA) and decreases HDL-C. In

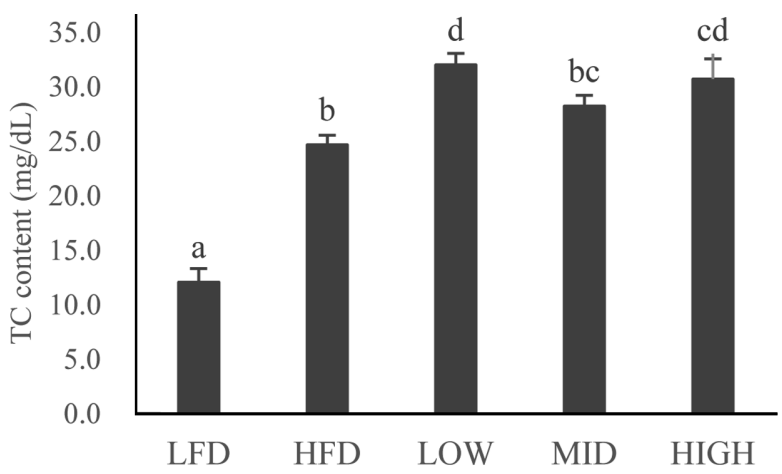

Fig. 6. Effect of fermented ginseng plus probiotic mixtures on TC content in feces.

${ }^{\text {abcd }}$ Means not sharing a common letter are significantly different at $p<0.05(n=8)$.

previous studies, ginseng [15] and probiotics [16] have been reported to improve the lipid profile by inhibiting hyperlipidemia. In the present study, there was no significant difference between TAG and HDL-C. In contrast, the TC was decreased in the HFD groups and increased in the FG+PM groups, with the exception of the LOW group. The biosynthesis of cholesterol is regulated by HMG-CoA reductase (HMGCR). Therefore, most cholesterol in a HFD inhibits HMGCR feed-back and prevents cholesterol biosynthesis. In the present study, FG+PM seemed to promote cholesterol biosynthesis through the positive feedback of HMGCR. In fact, previous studies have shown that ginsenoside Rb1 improved cholesterol biosynthesis [17]. Although it stimulated cholesterol biosynthesis, FG+PM also promoted the excretion of cholesterol into the feces (Fig. 6). Cholesterol can be converted into coprostanol by probiotics and then excreted directly into the feces, which decreases the amount of absorbed cholesterol [18]. However, both the control and the experimental groups in this study were within the normal cholesterol range; thus, it was difficult to state that dyslipidemia actually occurred.

Obesity causes hyperglycemia by counteracting FBG homeostasis. Obesity is associated with insulin resistance, and elevated FBG changes lipogenesis and lipolysis. According to the results of this study, although the FBG level was increased in the HFD group, the FG+PM level was lower and effectively inhibited in the MID group. This is consistent with the results that ginseng [15] and probiotics [19] decreased blood sugar. In fact, ginsenoside $\mathrm{Rd}$ has been shown to enhance glucose utilization through the PI-3 kinase-dependent pathway [20], and both ginsenosides Re and Rc have been reported to significantly 
increase glucose uptake in cells through AMPK activation [21].

Because the liver is one of the major organs responsible for lipid metabolism, damage to hepatic lipid metabolism leads to an abnormal accumulation of lipids, which is called hepatic steatosis. HFD-induced obesity increases FFA uptake into the liver, which often leads to hepatic fat accumulation resulting in non-alcoholic fatty liver disease (NAFLD) [22]. Ginseng and probiotics are well known as NAFLD-ameliorating agents. The results of this study show that the liver weight and hepatic TAG content were effectively suppressed in the FG+PM groups, especially in the MID group. Moreover, the smallest lipid droplet size was observed in the MID group (Fig. 2A).

Excessive energy intake results in the fat storage processes of hypertrophy and hyperplasia, which are controlled by PPAR- $\gamma$ [23]. The expression of PPAR- $\gamma$ was decreased by $50 \%$ in the MID group compared with the HFD group ( $p=$ $0.15)$. The results of the present study are similar to those found in earlier experimental studies on ginseng [24] and probiotics [25].

LDLR in the liver is known to take up LDL-C in the blood, which is involved in blood lipid clearance. LDLR expression was upregulated in the FG+PM groups and consistent with the decreased plasma LDL-C level in the LOW group. The results of our study concur with those found in a previous study [26]. Thus, FG+PM intake may have a role in decreasing the LDL-C concentration in the blood.

NAFLD is strongly associated with hepatic insulin resistance, type 2 diabetes, and obesity. Although insulin is a major regulator of hepatic lipogenesis, glucose is also known to contribute to the coordinated regulation of carbohydrate and fat metabolism in the liver [27]. The expression of G6P, which is involved in gluconeogenesis, was lower in the experimental groups. Compared with the control group, the expression of G6P in the FG+PM groups was lower, especially in the HIGH group by $90 \%(p=0.1)$. In contrast, the expression of GK, a glycolytic enzyme, was significantly elevated in the HFD group compared with the LFD group, which has the potential to promote the longterm storage of carbohydrates and TAG.

Taken together, this FG+PM combination effectively inhibited hepatic weight gain, TAG content, and liver fat accumulation, especially in the MID group.

Obesity is associated with hypertrophy in AT, which can cause adipocytes to move away from blood capillaries and result in adipocyte hypoxia [28]. Dead adipocytes form crown-like structures (CLS), and most macrophages that infiltrate the AT are found around the CLS [29]. AT macrophages promote insulin resistance and AT inflammation [30]. As found in previous studies [31, 32], FG+PM significantly suppressed the adipocyte size. In addition, FG+PM significantly inhibited the expression of TNF- $\alpha$ in the FG+PM groups (Fig. 5A), suggesting that FG+PM can inhibit hypertrophy by preventing the excessive formation of CLS in AT. In fact, Kim [33] reported that ginsenoside Re inhibited the expression of TNF- $\alpha$ in 3T3-L1.

PPAR- $\gamma$ is involved in the regulation of genes involved in lipid accumulation and insulin sensitivity [34]. PPAR- $\gamma$ is also used as a major target in the treatment of insulin resistance with thiazolidinediones (TZDs), PPAR- $\gamma$ activators. TZDs increase lipid accumulation in AT and decrease hepatic and muscular fat, ultimately inhibiting the fatty liver [35]. We observed that the MID group had an increased expression of PPAR- $\gamma$ compared with the control group ( $p=0.14$ ), which shows that FG+PM may have a role similar to TZDs. PPAR- $\gamma$ activation is associated with the expression of LPL, and fatty acid synthetase (FAS). LPL carries fat and degrades lipoprotein, which was approximately $50 \%$ higher in the MID group than in the control group $(p=0.15)$. The upregulation of LPL lowered FFA in the circulation and liver by increasing the fat inflow into AT, which decreased the hepatic lipotoxicity and improved insulin sensitivity [36]. The results of our study correspond well with those found in earlier studies showing that ginsenoside Re, Rg1, and Rb2 increase LPL expression [37], and ginsenosides $\mathrm{Rd}$ and $\mathrm{Rb} 1$ promoted adipose differentiation and lipid accumulation in 3T3-L1 cells [38]. FAS expression was suppressed by the consumption of FG+PM, especially in the HIGH group compared with the control group $(p=0.05)$. Therefore, FG+PM can improve insulin sensitivity through PPAR- $\gamma$ activation and inhibit fatty liver development. Thus, FG+PM exhibited an antiobesity effect.

In summary, the combination of fermented ginseng, B. longum BORI, and L. paracasei CH88 efficiently suppressed weight gain, hepatic and adipogenic fat accumulation, and TNF- $\alpha$ secretion in AT. This combination also improved the FBG level and increased cholesterol excretion into the feces. It is suggested that the FG+PM mixture could have an anti-obesity effect in HFD-fed mice.

\section{Acknowledgments}

This work was carried out with the support of the Promoting Regional Specialized Industry (R0004140), the Ministry of Trade, Industry and Energy (MOTIE) and 
Korea Institute for Advancement of Technology (KIAT), the Technological Innovation R\&D Program (Project No. S2463318) funded by the Small and Medium Business Administration (SMBA, Korea), and the Bio \& Medical Technology Development Program of the National Research Foundation (NRF) funded by the Korean MSIT (Project No. NRF-2017M3A9F3041747), Republic of Korea. The authors wish to thank Dr. Seockmo Ku of Middle Tennessee State University for his review and feedback on this paper.

\section{Conflict of Interest}

The authors have no financial conflicts of interest to declare.

\section{References}

1. Jung S, Lee M-S, Shin Y, Kim C-T, Kim I-H, Kim YS, et al. 2014. Anti-obesity and anti-inflammatory effects of high hydrostatic pressure extracts of ginseng in high-fat diet induced obese rats. J. Funct. Foods 10: 169-177.

2. Ku S, Park MS, Ji G, You H. 2016. Review on Bifidobacterium bifidum BGN4: functionality and nutraceutical applications as a probiotic microorganism. Int. J. Mol. Sci. 17: E1544.

3. Shen J, Obin MS, Zhao L. 2012. The gut microbiota, obesity and insulin resistance. Mol. Aspects Med. 34: 39-58.

4. Park MS, Kwon B, Ku S, Ji GE. 2017. The efficacy of Bifidobacterium longum BORI and Lactobacillus acidophilus ad031 probiotic treatment in infants with rotavirus infection. Nutrients 9: E887.

5. Li Z, Jin H, Oh SY, Ji GE. 2016. Anti-obese effects of two lactobacilli and two bifidobacteria on ICR mice fed on a high fat diet. Biochem. Biophys. Res. Commun. 480: 222-227.

6. Ku S. 2016. Finding and producing probiotic glycosylases for the biocatalysis of ginsenosides: a mini review. Molecules 21: 645.

7. Ku S, You H, Park MS, Ji G. 2016. Whole-cell biocatalysis for producing ginsenoside $\mathrm{Rd}$ from $\mathrm{Rb} 1$ using Lactobacillus rhamnosus GG. J. Microbiol. Biotechnol. 26: 1206-1215.

8. Ku S, You H, Park M, Ji G. 2015. Effects of ascorbic acid on $\alpha$-l-arabinofuranosidase and $\alpha$-l-arabinopyranosidase activities from Bifidobacterium longum RD47 and its application to whole cell bioconversion of ginsenoside. J. Korean Soc. Appl. Biol. Chem. 58: 857-865.

9. Karikura M, Miyase T, Tanizawa H, Takino $\mathrm{Y}$, Taniyama T, Hayashi T. 1990. Studies on absorption, distribution, excretion and metabolism of ginseng saponins. V. The decomposition products of ginsenoside $\mathrm{Rb} 2$ in the large intestine of rats. Chem. Pharm. Bull. 38: 2859.

10. Ouwehand AC, Kirjavainen PV, Shortt C, Salminen S. 1999. Probiotics: mechanisms and established effects. Int. Dairy J. 9: 43-52.
11. Folch J, Lees M, Sloane Stanley GH. 1957. A simple method for the isolation and purification of total lipides from animal tissues. J. Biol. Chem. 226: 497.

12. Thaler JP, Schwartz MW. 2010. Inflammation and obesity pathogenesis: the hypothalamus heats up. J. Clin. Endocrinol. Metab. 95: 4077.

13. Liu W, Zheng Y, Han L, Wang H, Saito M, Ling M, et al. 2008. Saponins (ginsenosides) from stems and leaves of Panax quinquefolium prevented high-fat diet-induced obesity in mice. Phytomedicine 15: 1140-1145.

14. Yoo SR, Kim YJ, Park DY, Jung UJ, Jeon SM, Ahn YT, et al. 2013. Probiotics L. plantarum and L. curvatus in combination alter hepatic lipid metabolism and suppress diet-induced obesity. Obesity 21: 2571-2578.

15. Yun S, Moon S, Ko S, Im B, Chung S. 2004. Wild ginseng prevents the onset of high-fat diet induced hyperglycemia and obesity in ICR mice. Arch. Pharm. Res. 27: 790-796.

16. Zhang X-L, Wu Y-F, Wang Y-S, Wang X-Z, Piao C-H, Liu J-M, et al. 2017. The protective effects of probiotic-fermented soymilk on high-fat diet-induced hyperlipidemia and liver injury. J. Funct. Foods 30: 220-227.

17. Sakakibara K, Shibata Y, Higashi T, Sanada S, Shoji J. 1975. Effect of ginseng saponins on cholesterol metabolism. I. The level and the synthesis of serum and liver cholesterol in rats treated with ginsenosides. Chem. Pharm. Bull. 23: 1009.

18. Lye HS, Rusul G, Liong MT. 2010. Removal of cholesterol by lactobacilli via incorporation and conversion to coprostanol. J. Dairy Sci. 93: 1383-1392.

19. Andersson U, Bränning $C$, Ahrné $S$, Molin G, Alenfall J, Önning G, et al. 2010. Probiotics lower plasma glucose in the high-fat fed C57BL/6J mouse. Benef. Microbes 1: 189-196.

20. Park S, Ahn IS, Kwon DY, Ko BS, WK Jun. 2008. Ginsenosides $\mathrm{Rb} 1$ and $\mathrm{Rg} 1$ suppress triglyceride accumulation in 3T3-L1 adipocytes and enhance $\beta$-cell insulin secretion and viability in Min6 cells via PKA-dependent pathways. Biosci. Biotechnol. Biochem. 72: 2815-2823.

21. Hwang JT, Lee M, Kim M, Kwon DY. 2008. Biological active components found in Panax ginseng improve glucose uptake via AMPK signaling pathway. FASEB J. 22: 683.

22. Dowman JK, Tomlinson JW, Newsome PN. 2010. Pathogenesis of non-alcoholic fatty liver disease. QJM. 103: 71-83.

23. Zhang L, Virgous C, Si H. 2017. Ginseng and obesity: observations and understanding in cultured cells, animals and humans. J. Nutr. Biochem. 44: 1-10.

24. Zhang Y, Yu L, Cai W, Fan S, Feng L, Ji G, Huang C. 2014. Protopanaxatriol, a novel PPAR $\gamma$ antagonist from Panax ginseng, alleviates steatosis in mice. Sci. Rep. 4: 7375.

25. Wu C-C, Weng W-L, Lai W-L, Tsai H-P, Liu W-H, Lee M-H, et al. 2015. Effect of strain K21 on high-fat diet-fed obese mice. Evid. Based Complement. Alternat. Med. 2015: 391767.

26. Park Y-H, Kim J-G, Shin Y-W, Kim S-H, Whang K-Y. 2007. Effect of dietary inclusion of Lactobacillus acidophilus ATCC 43121 on cholesterol metabolism in rats. J. Microbiol. Biotechnol. 
17: 655-662.

27. Dentin R, Girard J, Postic C. 2005. Carbohydrate responsive element binding protein (ChREBP) and sterol regulatory element binding protein-1c (SREBP-1c): two key regulators of glucose metabolism and lipid synthesis in liver. Biochimie 87: 81-86.

28. Crandall DL, Goldstein BM, Huggins F, Cervoni P. 1984. Adipocyte blood flow: influence of age, anatomic location, and dietary manipulation. Am. J. Physiol. 247: R46-R51.

29. Murano I, Barbatelli G, Parisani V, Latini C, Muzzonigro G, Castellucci M, Cinti S. 2008. Dead adipocytes, detected as crown-like structures, are prevalent in visceral fat depots of genetically obese mice. J. Lipid Res. 49: 1562.

30. Strissel KJ, Stancheva Z, Miyoshi H, Perfield JW, Defuria J, Jick Z, et al. 2007. Adipocyte death, adipose tissue remodeling, and obesity complications. Diabetes 56: 2910.

31. Lee H, Park D, Yoon M. 2013. Korean red ginseng (Panax ginseng) prevents obesity by inhibiting angiogenesis in high fat diet-induced obese C57BL/6J mice. Food Chem. Toxicol. 53: 402-408.

32. Takemura N, Okubo T, Sonoyama K. 2010. Lactobacillus plantarum strain no. 14 reduces adipocyte size in mice fed high-fat diet. Exp. Biol. Med. 235: 849-856.

33. Kim SO. 2007. Ginseng saponin-Re and Coix lachrymajobi var. mayuen regulate obesity related genes expressions, TNF-alpha, leptin, lipoprotein lipase and resistin in 3T3-L1 adipocytes. J. Life Sci. 17: 1523-1532.

34. Oh J, Lee H, Park D, Ahn J, Shin SS, Yoon M. 2012. Ginseng and its active components ginsenosides inhibit adipogenesis in 3T3-L1 cells by regulating MMP-2 and MMP-9. Evid. Based Complement. Alternat. Med. 2012: 265023.

35. Ciaraldi TP, Kong AP, Chu NV, Kim DD, Baxi S, Loviscach M, et al. 2002. Regulation of glucose transport and insulin signaling by troglitazone or metformin in adipose tissue of type 2 diabetic subjects. Diabetes 51: 30-36.

36. Choi S-S, Park J, Choi JH. 2014. Revisiting PPAR $\gamma$ as a target for the treatment of metabolic disorders. BMB Rep. 47: 599.

37. Masuno H, Kitao H, Okuda H. 1996. Ginsenosides increase secretion of lipoprotein lipase by 3T3-L1 adipocytes. Biosci. Biotechnol. Biochem. 60: 1962-1965.

38. Sekiya K, Okuda H, Hotta Y, Arichi S. 1987. Enhancement of adipose differentiation of mouse 3T3-L1 fibroblasts by ginsenosides. Phytother. Res. 1: 58-60. 